\title{
Holographic transmission gratings stored in a hydrogel matrix
}

Ramírez, Manuel, Lucío, María Isabel, Morales-Vidal, M., Beléndez, Augusto, Bañuls, María-José , et al.

Manuel G. Ramírez, María Isabel Lucío, M. Morales-Vidal, Augusto Beléndez, María-José Bañuls, Ángel Maquieira, Inmaculada Pascual, "Holographic transmission gratings stored in a hydrogel matrix," Proc. SPIE 11367,

Photosensitive Materials and their Applications, 113670B (1 April 2020); doi: 10.1117/12.2555362

SPIE. Event: SPIE Photonics Europe, 2020, Online Only, France 


\title{
Holographic transmission gratings stored in a hydrogel matrix
}

\author{
Manuel G. Ramírez ${ }^{\mathrm{a}, b^{*}}$, María Isabel Lucío ${ }^{\mathrm{d}}$, M. Morales-Vidal ${ }^{\mathrm{b}}$, Augusto Beléndez ${ }^{\mathrm{b}, \mathrm{c}}$, María-José \\ Bañuls $^{\mathrm{d}, \mathrm{e}}$, Ángel Maquieira ${ }^{\mathrm{d}, \mathrm{e}}$ and Inmaculada Pascual ${ }^{\mathrm{a}, \mathrm{b}}$ \\ ${ }^{a}$ Departamento de Óptica, Farmacología y Anatomía, Universidad de Alicante, Apartado de correos \\ 99, Alicante E-03080, Spain. \\ ${ }^{\text {b}}$ I.U. Física Aplicada a las Ciencias y las Tecnologías, Universidad de Alicante, Apartado de correos \\ 99, Alicante E-03080, Spain \\ 'Departamento de Física, Ingeniería de Sistemas y Teoría de la Señal, Universidad de Alicante, \\ Apartado de correos 99, Alicante E-03080, Spain. \\ ${ }^{\mathrm{d} I n s t i t u t o ~ I n t e r u n i v e r s i t a r i o ~ d e ~ I n v e s t i g a c i o ́ n ~ d e ~ R e c o n o c i m i e n t o ~ M o l e c u l a r ~ y ~ D e s a r r o l l o ~}$ \\ Tecnológico (IDM), Universitat Politècnica de València, Universitat de València, Camino de Vera \\ s/n, 46022 Valencia, Spain \\ 'Departamento de Química, Universitat Politècnica de València, Camino de Vera s/n, 46022 \\ Valencia, Spain
}

*Corresponding author: ramirez@ua.es

\begin{abstract}
The development and optimization of photopolymers in which time-stable holographic grating can be stored when the material is immersed in an aqueous medium represent a challenge at present. In this sense, the aim of this work was the fabrication of unslanted transmission gratings in a hydrogel matrix which incorporates in its molecular structure a monomer (2-Methacryloyloxyethyl phosphorylcholine) that can act as an anti-fouling agent. The dependence of the diffraction efficiency on the thickness of the hydrogel matrix and the stability of the transmission gratings immersed in water were also studied. An increment in the diffraction efficiency up to $47 \%$ was observed after a washing stage. After two days immersed in water, the holograms showed high stability even though the diffraction efficiency decreased to $37 \%$. The optical parameters were obtained by fitting procedure through Kogelnik's coupled wave theory.
\end{abstract}

Keywords: photopolymers, holographic transmission gratings, hydrogel, volume holography

\section{INTRODUCTION}

In past decades, several sensitive materials such as silver halide emulsions ${ }^{1}$, photoresists ${ }^{2}$, dichromate gelatine ${ }^{3}$, photorefractive materials ${ }^{4}$ and photopolymers ${ }^{5}$ have been used as recording materials in holography. In recent years, research has focused in photopolymers due to their excellent properties: chemical versatility for the design and preparation of different compositions, dry-processing availability, self-processing capabilities, large dynamic range, high diffraction efficiency and high resolution, good stability on thick emulsion layers, high sensitivity and relative low cost. These materials have many applications, such as fabrication of holographic waveguides ${ }^{6}$, holographic optical elements (HOEs) ${ }^{7}$, holographic memories ${ }^{8}$, non-image systems ${ }^{9}$ and holographic sensors ${ }^{10}$. Some features will be more important than others depending on the final application. A photopolymer is basically composed of an inert binder polymer, a sensitizing dye, an initiator, which is a free radical generator, and one or more polymerizable monomers. Poly(vinyl alcohol), polyacrylate and polyvinylchloride are examples of polymers used as binders. When the material is illuminated with a light at certain wavelength, the dye is excited and activates the initiator. Thus, free radicals are generated and combined with monomers which yields chain initiators. Therefore, in holography, the polymerization reaction occurs in the recording stage. During

Photosensitive Materials and their Applications, edited by Robert R. McLeod, Inmaculada Pascual Villalobos,

Yasuo Tomita, John T. Sheridan, Proc. of SPIE Vol. 11367, 113670B - (c) 2020 SPIE

CCC code: $0277-786 \mathrm{X} / 20 / \$ 21 \cdot$ doi: $10.1117 / 12.2555362$ 
the process of storing or recording a holographic grating in a photopolymer material, polymerized (exposed) and nonpolymerized (non-exposed) zones are generated due to the action of the laser source. At the same time, molecular diffusion processes are produced inside the photopolymers; that is, dye and monomer molecules diffuse from non-exposed to exposed zone and polymer chains diffuse out from the exposed zone towards the non-exposed zone. Thus, the hologram is not stable and the diffraction efficiency $(D E)$, i.e., the ratio between the power of diffracted and incident beams, decreases over time. A curing process is necessary to stop molecular diffusion processes and to provide temporal stability to holographic gratings. For that, different technics have been used such as exposure to ultraviolet light or incoherent light and dehydration of the photopolymer layer ${ }^{11-13}$.

Many investigations are carried out with the aim of developing photopolymer materials with improved properties. One of most important properties to consider is the stability of the holographic material in the medium in which it is supposed to work. The majority of photopolymers used in holography contains poly(vinyl alcohol), gelatin binders and monomers based in acrylamide or acrylate ${ }^{14,15}$. In these materials, the temporal stability of the holographic gratings is achieved through a curing stage carried out after the recording stage ${ }^{16}$ as their subsequent applications require that holograms are just preserved in ambient conditions, either in air or with a coating layer. However, other types of emerging applications require that holograms stored in materials are stable in an aqueous medium ${ }^{17}$. Taking into account that poly(vinyl alcohol), gelatin binders and monomers based in acrylamide or acrylate are water-soluble, it is necessary to develop and optimize other materials, such as hydrogels matrices, in which time-stable holograms can be stored when the material is immersed in an aqueous medium. This is a real challenge as, in these cases, the diffusion processes of the photopolymer components, which decrease the $D E$ values, can be more pronounced.

In this work, unslanted holographic transmission gratings were stored through the symmetrical experimental setup in a hydrogel matrix based in acrylamide with a spatial frequency of 1205 lines $/ \mathrm{mm}$ at recording wavelengths of $532 \mathrm{~nm}$. The curing stage was suppressed, and a washing step was carried out to eliminate the components that did not polymerize. 2Methacryloyloxyethyl phosphorylcholine was added in the mixture. Phosphorylcholine moieties are known to act as antifouling agents ${ }^{18}$. The $D E$ behaviour as function as the thickness of the hydrogels and the stability of the transmission gratings immersed in water were studied. An increase in the $D E$ was observed when the photopolymer layer was washed after the storing stage. With the aim to understand the behaviour of the $D E$, the Kogelnik's Coupled wave theory was used to fit the experimental values and to obtain the optical parameters.

\section{EXPERIMENTAL PROCEDURE}

\subsection{Material preparation}

All compounds were purchased from Sigma-Aldrich Química SL (Madrid, Spain). The preparation of the photopolymer material involves four stages. First, the functionalized hydrogel matrix incorporating the recognition component is prepared. A water solution composed of $89.9 \mathrm{~mol} \%$ acrylamide (AA), $3.4 \mathrm{~mol} \%$ N, $N^{\prime}$-Methylenebis(acrylamide) (MBA), $4.7 \mathrm{~mol} \%$ 2-Methacryloyloxyethyl phosphorylcholine (MPC) as bioactive recognition monomer and $1.2 \mathrm{~mol} \%$ potassium persulfate (KPS) and $0.7 \mathrm{~mol} \% N, N, N^{\prime}, N^{\prime}$-tetramethylethylenediamine (TEMED) as initiators was deposited through the force of gravity on levelled glass plates $(6.3 \times 6.3 \mathrm{~cm})$, which were previously washed and dried. Moulds with different heights $\left(h_{\mathrm{m}}\right)(80,160$ and $480 \mu \mathrm{m})$ were placed on these glass plates in order to obtain different thicknesses of the hydrogel matrices. Polymerization was carried out for 2 hours at room temperature. Then, hydrogels were washed with water. In the second stage, the hydrogel matrices were immersed for 3 hours in a solution containing $68.7 \mathrm{~mol} \% \mathrm{AA}, 7.8 \mathrm{~mol} \% \mathrm{BMA}$, $6.9 \mathrm{~mol} \%$ triethanolamine (TEA) and $16.5 \mathrm{~mol} \%$ yellow eosin (YE) as initiator dye. The process was carried out under controlled light conditions in which the material is not sensitive. After the third stage of holographic recording, a final stage of water washing was carried out for 30 minutes.

\subsection{Scanning electron microscopy characterization}

The hydrogel microstructures were analyzed by scanning electron microscopy (SEM) by using a Gemini SEM 500 system (Zeiss). First, hydrogels were completely swollen in water and frozen. Then, they were dried overnight in a Telstar Lyoquest freeze-drier to yield dry aerogel samples. Finally, SEM samples were prepared just before they were analyzed 
by sputter coating the dry aerogel samples with an Au layer of about $15 \mathrm{~nm}$ in a BAL-TEC SCD 005 sputter coater (Leica microsystems).

\subsection{Holographic transmission set-up}

Figure 1a shows the experimental holographic set-up to record and reconstruct unslanted transmission gratings in $100 \%$ humidity conditions. A continuous $(\mathrm{CW}) \mathrm{Nd}: \mathrm{YVO}_{4}$ laser emitting at $\lambda=532 \mathrm{~nm}$ was used. The material is sensitive for this wavelength as observed in Figure 2 where the transmittance of a photopolymer layer with a thickness of around 200 $\mu \mathrm{m}$ is shown. The transmission spectrum at normal incidence upon the photopolymer film was measured with a double beam spectrophotometer (V-650, Jasco). The beam laser was split into two secondary beams, the reference and object beams, using a beam-splitter. The ratio of intensities between both reference and object beams was 1.1 . Then, the beams were spatially filtered and collimated. The diameter of both beams was of $0.7 \mathrm{~cm}$. The laser beams have linear polarization perpendicular to the plane of incidence. This allows optimal interference since the interfering electric fields of the recording beams are parallel. The holographic transmission gratings were recorded by the appropriate interference between them. The object and reference beams were spatially overlapped at the sample with a recording angles $\theta_{o}=\theta_{r}=\theta=18.7^{\circ}$ with respect to the normal incidence. The working total intensity (sum of both intensity beams measures in the hologram plane) was $23.6 \mathrm{~mW} / \mathrm{cm}^{2}$. According to Bragg's law for symmetrical transmission geometry [equation (1)], theoretical spatial frequencies of $1205 \mathrm{lines} / \mathrm{mm}$ (period $\Lambda=0.829 \mu \mathrm{m}$ ) were obtained. Holographic transmitted grating illuminated over white light is shown in Figure 1b.

$$
\Lambda=\frac{\lambda}{2 \sin \theta}
$$

In the reconstruction stage, the diffracted and transmitted intensity were monitored in real-time with a He-Ne laser positioned at Bragg's angle $\left(\theta_{\text {Bragg }}=22.4^{\circ}\right)$ emitted at $632.8 \mathrm{~nm}$, at which the material is not sensitive, as it can be seen in Figure 2.

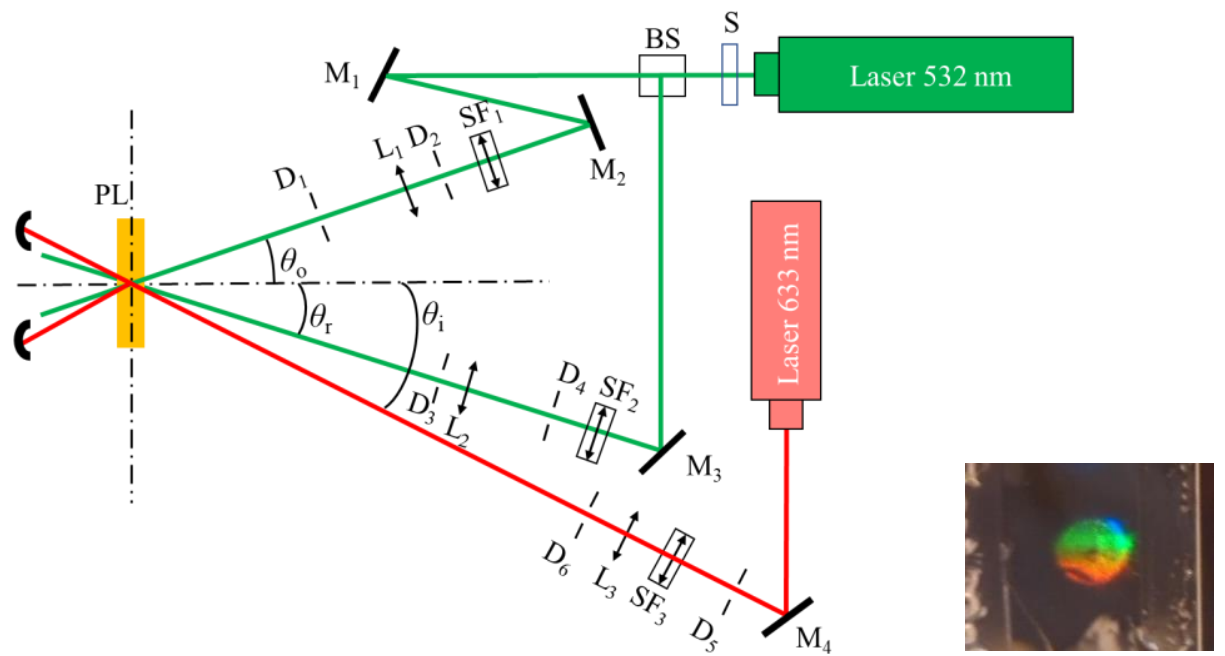

a)

b)

Figure 1. a) Holographic setup for transmission gratings. S: shutter; BS: beam splitter; $\mathrm{SF}_{\mathrm{i}}$ : spatial filters (microscope objective and pinhole); $\mathrm{M}_{\mathrm{i}}$ : mirrors; $\mathrm{L}_{\mathrm{i}}$ : lens; $\mathrm{D}_{\mathrm{i}}$ : diaphragms; $\theta_{0}, \theta_{\mathrm{r}}$ : object and reference recording angle, $\theta_{i}$ : incident reconstruction angle; PL: photopolymer layer. b) Holographic transmission grating illuminated over white light. 


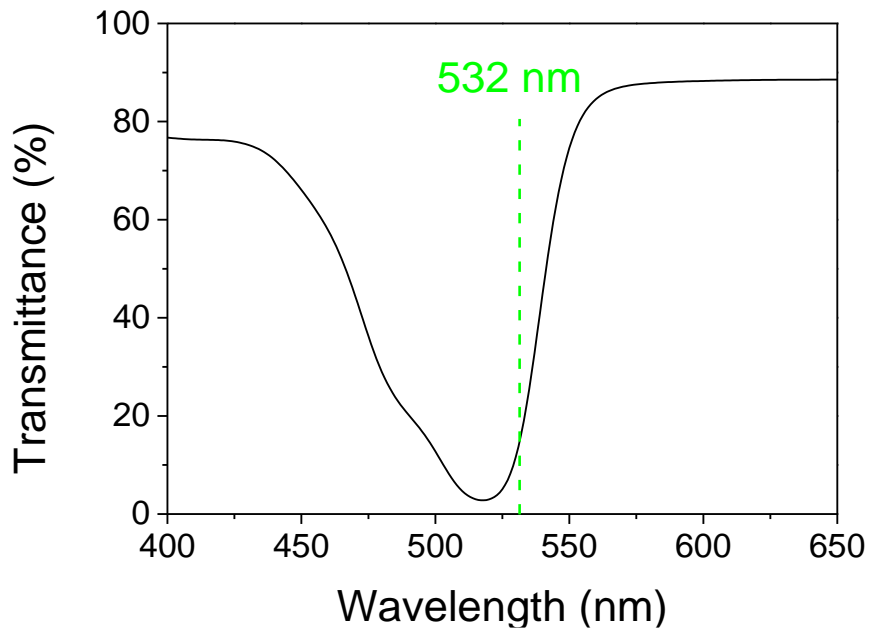

Figure 2. Transmission spectrum of a hydrogel matrix after being immersed in a solution containing AA, BMA, TEA and YE.

\subsection{Analysis of experimental data through Kogelnik's Coupled Wave Theory}

According to Kogelnik's Coupled wave theory ${ }^{19}$, the $D E$ of phase holographic transmission gratings is given by:

$$
\left.D E=e^{\left(-\alpha d / \cos \theta^{\prime}\right.}{ }_{i}\right) \frac{\sin ^{2} \sqrt{v^{2}+\xi^{2}}}{1+\frac{\xi^{2}}{v^{2}}}
$$

The experimental diffraction efficiency as a function of the Bragg deviation angle can be fitted by means of Kogelnik's Coupled wave theory [equation (2)]. Thus, the optical thickness $(d)$, refractive index modulation $(\Delta n), \Lambda$, refractive index $(n)$ and absorption loss coefficient $(\alpha)$ can be obtained. The parameter that controls $D E$ at Bragg condition is $v$ [Equation (3)], while the $\xi$ parameter [equation (4)] is related to the deviation from the exact Bragg condition.

$$
\begin{gathered}
\nu=\frac{\pi \Delta n d}{\lambda \sqrt{c_{r} c_{S}}} \\
\xi=\frac{\pi d}{\Lambda c_{S}}\left[\left|\sin \left(\theta^{\prime}{ }_{i}-\varphi\right)\right|-\frac{\lambda}{2 n \Lambda}\right]
\end{gathered}
$$

Slant factors $\left(c_{\mathrm{r}}\right.$ and $\left.c_{\mathrm{s}}\right)$ are defined in equation (5) and equation (6), respectively. The $\varphi$ parameter represented the fringe tip angles and $\theta_{i}^{\prime}$ is the incident reconstruction angle inside of the photopolymer layer.

$$
\begin{gathered}
c_{r}=\cos \theta^{\prime}{ }_{i} \\
c_{S}=\cos \theta^{\prime}{ }_{i}-\frac{\lambda}{n \Lambda} \sin \varphi
\end{gathered}
$$

\section{RESULTS AND DISCUSSION}

Figure $3 \mathrm{a}$ and $3 \mathrm{~b}$ show the digital images of the bulk hydrogel in its swollen state (zenith and lateral angles). The total transparency of the material can be observed in both images. Figure $3 \mathrm{c}$ shows the microscopic morphology of the same 
hydrogel studied by Scanning Electron Microscopy. In the SEM image a porous structure can be observed. This porous structure is necessary for the diffusion of the photopolymer components into hydrogel matrix during the hologram fabrication.

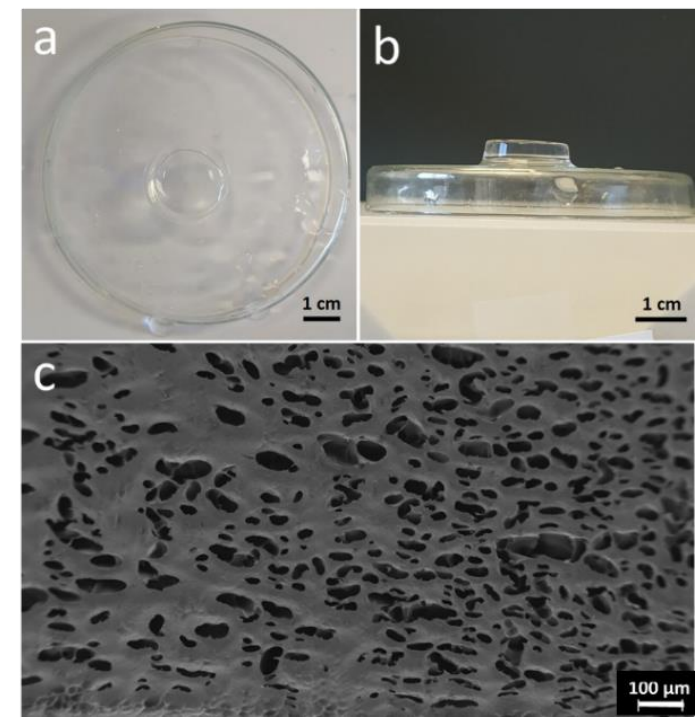

Figure 3. a) and b) Digital images and c) SEM image of the bulk hydrogel.

Figure 4 shows the experimental $D E$ values in percentage as a function of the reconstruction angle of three photopolymer layers obtained by depositing hydrogels matrices in moulds with three different heights $\left(h_{\mathrm{m}}=80,160\right.$ and $\left.480 \mu \mathrm{m}\right)$. The origin of the axes corresponds to the Bragg angle at the reconstruction wavelength. As it can be observed, a maximum of efficiency diffraction experimental values $\left(D E_{\max }\right)$ of around $17 \%$ is reached when $h_{\mathrm{m}}=160 \mu \mathrm{m}$. This $D E_{\max }$ value is similar to that obtained when $h_{\mathrm{m}}=80 \mu \mathrm{m}$. There is a noticeable decrease in $D E_{\max }$ value $(4 \%)$ when the $h_{\mathrm{m}}$ increases to $480 \mu \mathrm{m}$.



Figure 4. $D E$ as a function of the reconstruction angle for transmission gratings. The thickness of the moulds where the hydrogel matrix was deposited were 80 (black square), 160 (red circles) and $480 \mu \mathrm{m}$ (blue triangles). The spatial frequency and the total recording intensity were 1205 lines $/ \mathrm{mm}$ and $23.6 \mathrm{~mW} / \mathrm{cm}^{2}$, respectively. 
As already mentioned, the study of the stability of the transmission grating stored in a photopolymer material is essential. Once a $h_{\mathrm{m}}$ value of $160 \mu \mathrm{m}$ was selected as the optimal thickness of the hydrogel, the stability of the transmission grating immersed in water was investigated. For this, reconstruction measures were carried out at three different times. The results are shown in Figure 5. First, the reconstruction immediately after the recording stage (RS) was carried out and a $D E_{\max }$ value of $20 \%$ was obtained. When the washing stage (WS) was carried out following the procedure previously described in section 2.1, the $D E_{\max }$ increase up to $47 \%$. Then, the transmission grating was stored in water at $6{ }^{\circ} \mathrm{C}$ for 2 days. After this time, a $D E_{\max }$ value of $37 \%$ was measured.

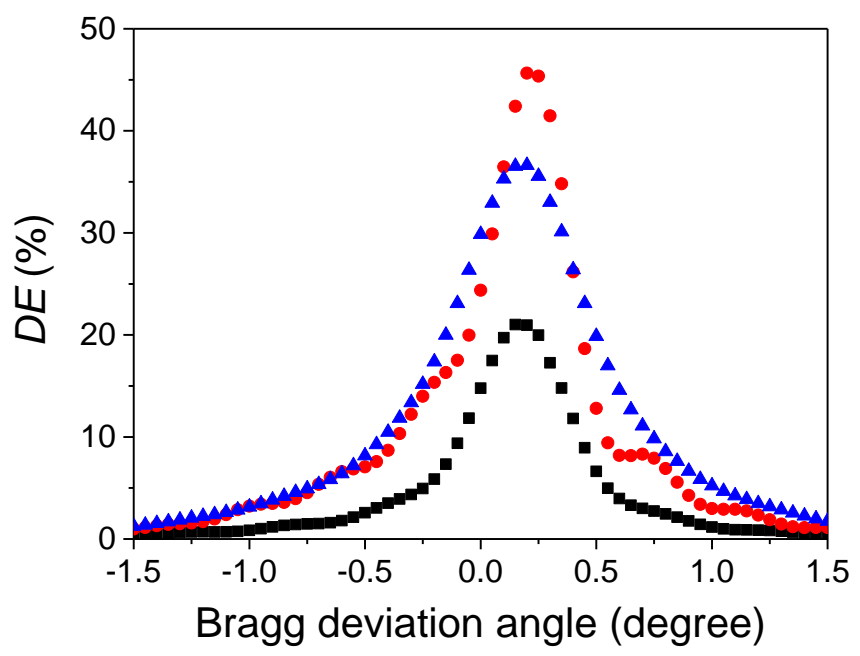

Figure 5. $D E$ as a function of the reconstruction angle for a transmission grating reconstructed immediately after the recording stage (black square), after washing (red circles) and after 2 days in water at $6{ }^{\circ} \mathrm{C}$ (blue triangles).

In order to understand the $D E_{\max }$ behaviour between both, reconstruction immediately after recording and after washing stages, a theoretical fit of the experimental data with the Kogelnik's Coupled wave theory [equation (3)] was carried out. The optical parameters $\alpha, n, \Lambda, \Delta n$ and $d$ were obtained from the fitting, which is represented with a solid line in Figure 6. The results obtained for all the parameters are shown in Table 1. The $D E_{\max }$ is calculated from fit. The $n$ values are the same for both stages while the $\alpha_{\mathrm{RS}}$ is around three times higher than $\alpha_{\mathrm{WS}}\left(\alpha_{\mathrm{RS}}\right.$ and $\alpha_{\mathrm{WS}}$ denoted the absorption loss coefficient obtained after recording and washing stages). We hypothesize that the decrease in the value of $\alpha$ after WS can be due to an increase in the transmittance of the material as the components of the photopolymer that have not been polymerized are drawn out of the photopolymer layer during this stage. The $\Delta n$ is one of the most important optical parameters in the $D E$ of a holographic grating. $\Delta n$ for RS $\left(\Delta n_{\mathrm{RS}}=1.76 \cdot 10^{-3}\right)$ is slightly smaller than $\Delta n$ for WS $\left(\Delta n_{\mathrm{WS}}=1.96 \cdot 10^{-3}\right)$. When the washing stage is carried out, the AA and BMA molecules diffuse from the unexposed zone to the solution in which the photopolymer layer is washed decreasing the $n$ value in these areas. Thus, considering that $\Delta n=n$ (exposed zone) - $n$ (unexposed zone), an increase of the $\Delta n$ is expected. Regarding the optical thickness, a $d_{\text {Ws }}$ higher than $d_{\mathrm{RS}}$ value is observed. This fact can be explained due to a dimensional change in the material that can occur during the washing stage. Polymer chains formed in the exposed zones may undergo slight stretching in the direction perpendicular to the photopolymer film without noticeable destruction in the gradient. From equation (4), the maximum $D E_{\max }$ is obtained at the Bragg condition (in which the parameter $\xi$ is 0 ). In this point, $D E$ is dominated by parameter $v$, which depends on the product $\Delta n \cdot d$. As observed in Table $1, \Delta n \cdot d$ obtained in WS is greater than $\Delta n \cdot d$ reached in RS. This behaviour explains the increase of the $D E_{\max }$ value when the washing stage is carried out. When the $D E$ of the transmission grating was measured after 2 days immersed in water, a decrease of $D E_{\max }$ up to $37 \%$ and an increase in the angular bandwidth are observed. In addition, the lateral lobes do not appear. These facts can be explained considering that the polymeric network formed in the recording stage not is not sufficiently crosslinked. However, there is no shift in the angular position of the $D E_{\max }$ indicating that the period of the grating does not change after 2 days immersed in water. 


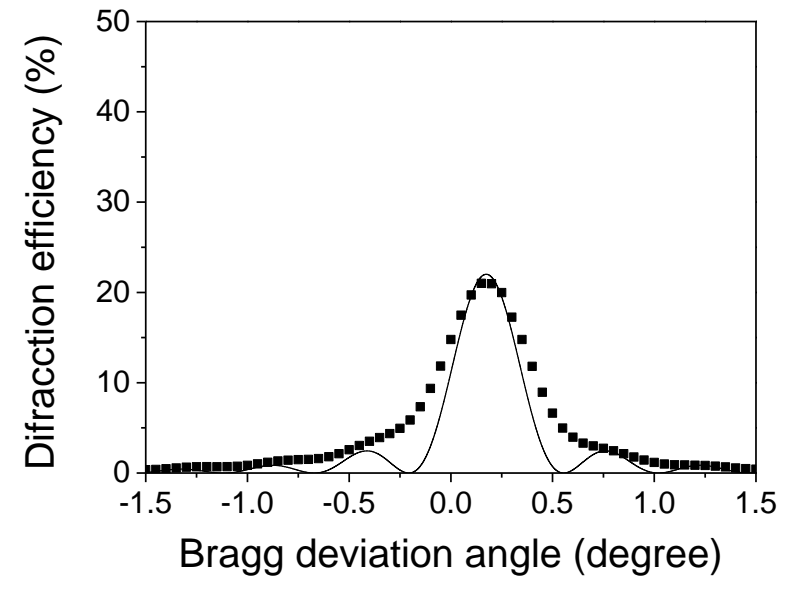

a)

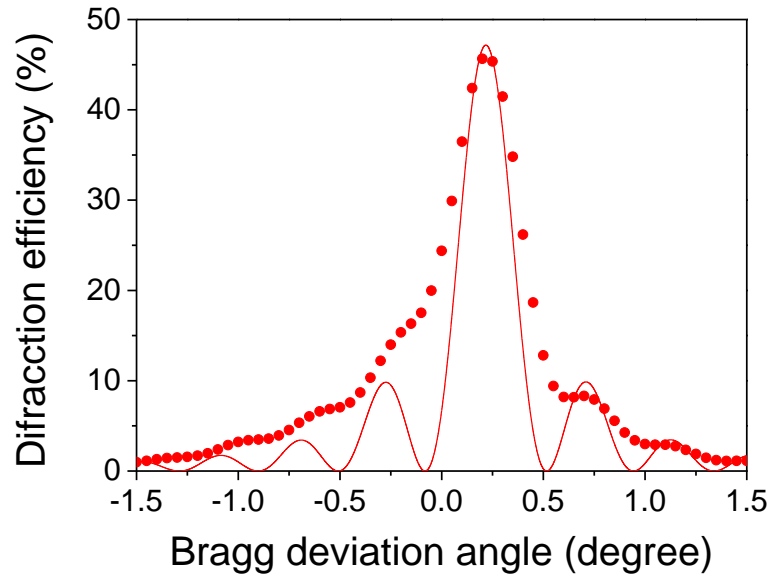

b)

Figure 6. $D E$ as a function of the reconstruction angle for a transmission grating a) immediately after the recording stage (RS) and b) after the washing stage (WS). Solid lines represent the theoretical fit through Kogelnik's Coupled wave theory.

Table 1. Parameters obtained from the theoretical fit of the experimental $D E$ values.

\begin{tabular}{|c|c|c|c|c|c|c|c|}
\hline Stage & $\boldsymbol{\alpha}\left(\boldsymbol{\mu \mathbf { m } ^ { - 1 } )}\right.$ & $\boldsymbol{n}$ & $\boldsymbol{\Lambda}(\boldsymbol{\mu m})$ & $\boldsymbol{\Delta n}$ & $\boldsymbol{d}(\boldsymbol{\mu m})$ & $\boldsymbol{\Delta n} \cdot \boldsymbol{d}(\boldsymbol{\mu m})$ & $\boldsymbol{D E} \max (\boldsymbol{\%})$ \\
\hline RS & 0.0086 & 1.500 & 0.824 & 0.00176 & 170 & 0.299 & 21.9 \\
\hline WS & 0.0030 & 1.500 & 0.823 & 0.00196 & 194 & 0.380 & 47.1 \\
\hline
\end{tabular}

\section{CONCLUSIONS}

In this work, transmission holographic gratings stored in a hydrogel matrix incorporating 2-Methacryloyloxyethyl phosphorylcholine have been shown. A washing stage in water increases the $D E_{\max }$ achieved in the recording stage until $47 \%$. After two days immersed in water, the $D E_{\max }$ decrease until $37 \%$ and there is no shift in the angular position of the $D E_{\max }$. Although more work is needed to optimize the photopolymer material, the results obtained show that the transmission grating retains great temporal stability in water.

\section{ACKNOWLEDGEMENTS}

Work funded by Ministerio de Ciencia e Innovación (Spain) under project FIS2017-82919-R (MINECO/AEI/FEDER, UE); by Generalitat Valenciana (Spain) under project CDEIGENT/2018/024; by the E.U. FEDER, the Spanish Ministry of Economy and Competitiveness under project MINECO (BiHolog-CTQ2016-75749-R). M. I. Lucío acknowledges MINECO for her Juan de la Cierva-formación grant (FJCI-2016-29593). M. I. Lucío and I. Pascual acknowledge the Programa Propio para el fomento de la I+D+I del Vicerrectorado de Investigación y Transferencia de Conocimiento of the Universidad de Alicante for the grant "Ayudas para estancias de personal investigador invitado " (INV19-15). 


\section{REFERENCES}

[1] Kim, J. M., Choi, B. S., Choi, Y. S., Kim, J. M., Bjelkhagen, H. I. and Phillips, N. J., "Holographic optical elements recorded in silver halide sensitized gelatin emulsions Part 2 Reflection holographic optical elements," Appl. Opt. 41(8), 1522 (2002).

[2] Pascual, I., Beléndez, A., Mateos, F. and Fimia, A., "Comparison between direct method and the copying method to obtain in HOEs in AZ-1350 photoresist material," Ópt. Pura Apl. 24, 63-67 (1991).

[3] Kubota, T. and Ose, T., "Lippmann color holograms recorded in methylene-blue-sensitized dichromated gelatin,” Opt. Lett. 1, 289-291 (1979).

[4] Gunter, P. and Huignard, J. P., "Photorefractive Materials and their Applications 3," K. Buse, F. Havermeyer, W. Liu, C. Moser, and D. Psaltis, Eds., Springer, New York, NY, 295-317 (2007).

[5] Close, D. H., Jacobson, A. D., Margerum, J. D., Brault, R. G. and McClung, F. J., "Hologram recording on photopolymer materials," Appl. Phys. Lett. 14(5), 159-160 (1969).

[6] Fernández, R., Bleda, S., Gallego, S., Neipp, C., Márquez, A., Tomita, Y., Pascual, I. and Beléndez, A., "Holographic waveguides in photopolymers," Opt. Express 27(2), 827-840 (2019).

[7] Lloret, T., Navarro-Fuster, V., Ramírez, M. G., Ortuño, M., Neipp, C., Beléndez, A. and Pascual, I., "Holographic lenses in an environment-friendly photopolymer," Polymers (Basel). 10(3) (2018).

[8] Fernández, E., Ortuño, M., Gallego, S., Márquez, A., García, C., Beléndez, A. and Pascual, I., "Multiplexed holographic data page storage on a polyvinyl alcohol/acrylamide photopolymer memory," Appl. Opt. 47(25), 4448-4456 (2008).

[9] Bañares-Palacios, P., Álvarez-Álvarez, S., Marín-Sáez, J., Collados, M.-V., Chemisana, D. and Atencia, J., "Broadband behavior of transmission volume holographic optical elements for solar concentration," Opt. Express 23(11), A671 (2015).

[10] Yetisen, A. K., Naydenova, I., Da Cruz Vasconcellos, F., Blyth, J. and Lowe, C. R., "Holographic sensors: Three-dimensional analyte-sensitive nanostructures and their applications," Chem. Rev. 114(20), 10654-10696 (2014).

[11] Navarro-Fuster, V., Ortuño, M., Fernández, R., Gallego, S., Marquez, A., Beléndez, A. and Pascual, I., "Peristrophic multiplexed holograms recorded in a low toxicity photopolymer," Procceding SPIE Pract. Hologr. VIII 7(1), 133-147, San Jose, CA, United States (2017).

[12] Jenney, J. A., "Holographic Recording with Photopolymers,” J. Opt. Soc. Am. 60, 1155-1161 (2008).

[13] Tipton, D. F., Armstrong, M. L. and Stevenson, S. H., "Improved process of refrection holography replication and heat processing,” Procceding IS\&T/SPIE 1994 Int. Symp. Electron. Imaging Sci. Technol., 172-183, San Jose, CA, USA (1994).

[14] Gallego, S., Ortuño, M., Neipp, C., Márquez, A., Beléndez, A. and Pascual, I., "Characterization of polyvinyl alcohol/acrylamide holographic memories with a first-harmonic diffusion model," Appl. Opt. 44(29), 6205-6210 (2005).

[15] Ortuño, M., Gallego, S., García, C., Pascual, I., Neipp, C. and Beléndez, A., "Holographic Characteristics of an Acrylamide/Bisacrylamide Photopolymer in 40-1000 $\mu \mathrm{m}$ Thick Layers," Phys. Scr. 2005, T118 (2005).

[16] Fernández, E., Fuentes, R., Ortuño, M., Beléndez, A. and Pascual, I., "Holographic grating stability: Influence of 4,4'-azobis (4-cyanopentanoic acid) on various spatial frequencies," Appl. Opt. 52, 6322-6331 (2013).

[17] Yetisen, A. K., Butt, H., Volpatti, L. R., Pavlichenko, I., Humar, M., Kwok, S. J. J., Koo, H., Kim, K. S., Naydenova, I., Khademhosseini, A., Hahn, S. K. and Yun, S. H., "Photonic hydrogel sensors," Biotechnol. Adv. 34, 250-271 (2016).

[18] Díaz-Betancor, Z., Bañuls, M. J., Sanza, F. J., Casquel, R., Laguna, M. F., Holgado, M., Puchades, R. and Maquieira, Á., "Phosphorylcholine-based hydrogel for immobilization of biomolecules. Application to fluorometric microarrays for use in hybridization assays and immunoassays, and nanophotonic biosensing," Microchim. Acta 186, 570 (2019).

[19] Kogelnik, H., "Coupled Wave Theory for Thick Hologram Gratings," Bell Syst. Tech. J. 48(9), 2909-2947 (1969). 